\title{
A PRÁXIS DO ENFERMEIRO NO PROGRAMA SAÚDE DA FAMÍLIA NA ATENÇÃO À SAÚDE MENTAL*
}

[The nurse's praxis in the family health program in attention to mental health care]

\author{
Khívia Kiss Barbosa de Sousa** \\ Maria de Oliveira Ferreira Filha*** \\ Ana Tereza Medeiros Cavalcanti da Silva****
}

RESUMO: Estudo realizado com objetivo de descrever as concepções dos enfermeiros que atuam no Programa Saúde da Família - PSF do município de Cabedelo sobre o processo de trabalho de enfermagem, as características desse processo, e, identificar os aspectos do processo saúde-doença que estes enfermeiros abordam com maior e menor freqüência, no cotidiano de sua prática profissional. Teve referencial teórico nos modelos de assistência à saúde implantados no Brasil, na estratégia do PSF, e, no processo de Trabalho em saúde mental. O referencial metodológico teve inspiração no Materialismo Histórico e Dialético. O material empírico foi coletado por meio de entrevista e analisado através da técnica de análise do discurso. A análise permitiu identificar os saberes e as práticas que orientam o trabalho investigado, auxiliando os sujeitos da investigação a desenvolver ações de enfermagem capazes de subsidiar uma aproximação com a superação das contradições reveladas, para adensar a construção de uma práxis, requerida na atualidade, que responda às necessidades de atenção à saúde mental, no

\footnotetext{
*Dissertação de Mestrado. Programa de Pós Graduação em Enfermagem da Universidade Federal da Paraíba/Brasil

${ }^{* *}$ Mestranda do Programa de Pós Graduação em Enfermagem da Universidade Federal da Paraíba.

${ }^{* * *}$ Doutora em Enfermagem. Profa. do Departamento de Enfermagem de Saúde Publica e Psiquiatria/Programa de Pós Graduação em

Enfermagem da Universidade Federal da Paraíba.

${ }_{* * * \star}$ Doutora em Enfermagem. Profa. do Departamento de Enfermagem de Saúde Publica e Psiquiatria/Programa de Pós Graduação em

Enfermagem da Universidade Federal da Paraíba.
}

sentido da inclusão social da pessoa com doença mental. O PSF é uma estratégia para a efetivação do Sistema Único de Saúde - SUS e como tal, um instrumento que pode ser empregado no sentido da municipalização das ações de saúde mental, aproximando essa área específica com o SUS e permitindo o avanço do movimento pela Reforma Psiquiátrica na perspectiva de uma de suas diretrizes, a desinstitucionalização da doença e da pessoa com doença mental.

PALAVRAS-CHAVE: Enfermagem, PSF, Saúde Mental.

\section{INTRODUÇÃO}

Este estudo aborda a práxis do enfermeiro no cuidado a saúde mental, no contexto do Programa Saúde da Família - PSF, tendo como perspectiva a efetivação do processo de Reforma da assistência psiquiátrica em curso no país, entendida como um projeto da política nacional de saúde mental do Ministério da Saúde - MS. Nessa perspectiva, a práxis do enfermeiro é compreendida como saberes e práticas que devem se objetivar em um novo processo de trabalho de base comunitária, por meio do PSF, estratégia do Sistema Único de Saúde - SUS.

O Sistema de Saúde no Brasil tem uma trajetória político ideológica, que reflete o desenvolvimento histórico, econômico e político da sociedade brasileira. Iniciado com o Sanitarismo Campanhista, na década de 1930, esse sistema 
organizou-se no enfoque médico assistencial privatista, instituído a partir da década de 1960, e após sucessivas críticas feitas pelo Movimento Sanitarista, na década de 1990 foi criado o Sistema Único de Saúde - SUS, atualmente em vigência no país, pautado nos princípios da universalidade, equidade e integralidade.

A universalidade pressupõe direitos iguais para todas as pessoas na atenção à saúde, cabendo ao Estado garantir as condições para o exercício desse direito. A equidade refere-se à garantia de acesso às ações e serviços para todos os cidadãos, em todo o país, diferenciando a assistência de acordo com a necessidade da população. A integralidade pressupõe que o sistema deve garantir, a todos os cidadãos, os serviços que forem necessários, abrangendo ações de promoção, prevenção, proteção, tratamento e recuperação da saúde, assistindo indivíduo, a família e a comunidade. O SUS é portanto, um sistema universal, equânime e integral (BRASIL, 1994).

Dentre os princípios do SUS a integralidade, vem sendo perseguida como a idéia transformadora do modelo de assistência. Ela também é compreendida como um cuidado que não concebe a idéia de disjunção mente-corpo, ainda presente, na prática dos trabalhadores da saúde que atuam no SUS. Para Mattos (2001) a integralidade se apresenta como um princípio de organização contínua do processo de trabalho nos serviços de saúde, através de uma busca permanente e ampliada que visa apreender as necessidades do grupo populacional, manifestando a compreensão de que os eventos de adoecimento não são apenas biológicos, mas também históricos, sociais, culturais e econômicos, com repercussões na existência das pessoas.

No enfoque da saúde mental o enfermeiro deve considerar como objeto do trabalho o sofrimento das pessoas e como finalidade a promoção da saúde, a prevenção de agravos à saúde mental, e a inclusão do portador de transtorno mental na vida comunitária.

Considerando a perspectiva da efetivação da reforma psiquiátrica é preciso considerar que a assistência à saúde mental articula-se com o modo de produção dos serviços de saúde, organizados em sistemas financeiros, materiais e de recursos humanos, articulados com a força de trabalho e com a tecnologia produzida pelos saberes e práticas, ou seja, pela práxis, termo definido por Konder (1992, p. 115) como "a ação que, para se aprofundar de maneira mais conseqüente, precisa de reflexão, do autoquestionamento, da teoria; e é a teoria que remete à ação, que enfrenta o desafio de verificar seus acertos e desacertos cotejando-os com a prática".

Conforme Torrez, Barros e Goulart (2000, p. 68-9) o Ministério da Saúde tem procurado estimular o desenvolvimento de "um novo processo de trabalho nos cuidados à saúde, substituindo as práticas convencionais de atendimento e de funcionamento das unidades de saúde", através do PSF. Este consiste em uma estratégia do SUS, que visa a atenção primária à saúde, na qual a pessoa deve ser vista como um ser não-divisível, como alguém necessitando que o seu processo saúde-doença seja compreendido na inter-relação das forças sociais, econômicas, históricas e culturais. A família deve ser abordada de modo integral e articulado e não mais de modo fragmentado. Essa nova estratégia fundamentase em uma prática interdisciplinar entre os profissionais de distintas categorias da saúde e de áreas afins.

O modelo assistencial em saúde é uma abstração que sintetiza a compreensão de uma categoria apoiada em três pilares articulados: as políticas de saúde, os serviços e o trabalho. As políticas de saúde podem ser compreendidas como um conjunto de intenções que orientam para a constituição e organização dos serviços de saúde, e estes se definem como o lugar geopolítico onde se processa o trabalho. O trabalho por sua vez se destaca por seu potencial transformador da realidade, efetivando-se por meio de uma processualidade (SILVA, 2003).

Os processos de trabalho organizam-se em uma sucessão de momentos essenciais à sua efetivação, a partir da definição de seus elementos, quais sejam: o objeto, a finalidade e os instrumentos. Esses elementos, por sua vinculação histórica e ideológica nas sociedades capitalistas, são legitimados pelas práticas quando agregam novos significados. Assim sendo, as ações de saúde mental no PSF concretizam-se a partir do reconhecimento do trabalho como uma categoria potente para responder às necessidades sociais 
que demandam transformações na assistência psiquiátrica tradicional.

Nesse momento histórico, os problemas que mais atingem a saúde da população estão relacionados com questões de âmbito social. $\mathrm{O}$ desemprego entre jovens e adultos, o abandono de crianças e idosos, a violência de todos os tipos, o alcoolismo e a dependência de substâncias químicas, a migração intra e inter urbana, gerando perdas de vínculos afetivos e sociais e, conseqüentemente, sofrimento psíquico e emocional que demandam a assistência em saúde mental e aproximam essa área específica do conhecimento com a saúde de uma maneira geral e informam a necessidade de se relacionar às questões de saúde, conhecimentos da área das Ciências Sociais.

Sendo o PSF uma estratégia para a municipalização dos serviços de saúde e, portanto, para a efetivação das ações demandadas pelo SUS e, entendendo que o trabalho de saúde mental é um dos pilares de sustentação da categoria Modelo de Assistência das mais importantes por seu potencial transformador da realidade, sentimos a necessidade de conhecer a práxis do enfermeiro no Programa de Saúde da Família.

Tendo em vista que o município de Cabedelo/ PB demonstrou interesse político no sentido de viabilizar um sistema de cuidado à saúde mental, através das equipes do PSF e, havendo por parte dos trabalhadores e da população uma expectativa no que diz respeito a mudanças que correspondam à reforma psiquiátrica, proposta no país, buscamos investigar o significado que assumem os processos de trabalho realizados pelos enfermeiros das equipes do PSF, na atualidade. Esse interesse motivou a elaboração das seguintes questões para nortear a investigação: Quais as concepções dos enfermeiros do PSF de Cabedelo sobre o seu processo de trabalho? Quais as características do processo de trabalho desenvolvido pelos enfermeiros do PSF do município de Cabedelo? Quais os aspectos do processo saúde doença que os enfermeiros abordam com maior e menor freqüência no cotidiano do seu processo de trabalho?

Essas questões foram suscitadas em momentos de aproximação com as equipes do
PSF no município de Cabedelo/PB, quando se discutiu com a coordenação da atenção básica de saúde da Secretaria Municipal a possibilidade da construção de uma rede de serviços de saúde mental, na qual as Unidades Básicas de Saúde do PSF seriam a porta de entrada do modelo de atenção a saúde mental e, portanto, teriam papel primordial na promoção da saúde, prevenção dos transtornos mentais e na inclusão social.

A perspectiva desse estudo foi a de contribuir para uma compreensão sobre a realidade de saúde mental do município de Cabedelo/PB, no sentido da produção de novos conhecimentos que subsidiem reflexões sobre a atenção básica em saúde mental. Buscou-se também elucidar para os enfermeiros, que as ações de saúde mental desenvolvidas na comunidade, devem estar pautadas por um novo processo, no qual a força de trabalho envolvida passe a organizar seu processo de trabalho a partir da concepção do processo saúde-doença mental, considerando também a existência-sofrimento das pessoas acometidas por doença mental. Esse exercício crítico é imprescindível para a construção de novas formas de conceber/assistir a pessoa em sofrimento psíquico.

\section{DESCRIÇÃO DA METODOLOGIA}

Neste estudo foi priorizada a abordagem qualitativa, pautada no referencial teórico o Materialismo Histórico e Dialético - MHD. Justificamos a escolha da abordagem porque ela busca compreender fatos não mensuráveis sobre o significado que o processo de trabalho de enfermagem no PSF revela, na perspectiva da Reforma Psiquiátrica, no município de Cabedelo $-\mathrm{PB}$.

A eleição metodológica do MHD justifica-se pela adequação de seus pressupostos e instrumentos ao objeto deste estudo. O método é constituído por uma teoria científica e crítica da história, o materialismo histórico, e por uma teoria filosófica, o materialismo dialético. Seu pressuposto é o de que nada é eterno, absoluto ou fixo. Tanto no mundo natural como no mundo social tudo está em permanente transformação e submetido ao movimento da história, como um processo temporal, movido por contradições cuja 
superação é mediada pelo trabalho (LOWY, 1989).

\section{Cenário do estudo}

Fundada no final do século XVI, na década de 1580, Cabedelo é uma cidade portuária e seu nome é derivado da expressão brasílica tupi que significa "pequeno cabo". Constitui-se em uma península entre o Oceano Atlântico e o Rio Paraíba, com área territorial de $31,42 \mathrm{Km}^{2}$. Está localizada ao norte do Estado da Paraíba. Tem seus limites, ao sul com o Rio Jaguaribe e o município de João Pessoa, capital do Estado; a oeste com o Rio Paraíba e com os municípios de Santa Rita e Lucena; a norte e leste com o Oceano Atlântico. Seu clima é quente e úmido, com temperatura máxima de $35^{\circ} \mathrm{C}$, e mínima de $22^{\circ} \mathrm{C}$. As chuvas começam geralmente em abril, e terminam em julho. Sua vegetação é composta de mata atlântica, coqueirais e manguezais.

A população do município perfaz um total de 48.417 habitantes (IBGE, 2000) onde $51 \%$ são mulheres e $49 \%$ são homens, sendo a concentração predominantemente urbana. A atividade principal é o comércio, de derivados de petróleo e os serviços de operação portuária; secundariamente, desenvolvem a pesca e o turismo. Vale ressaltar que no município encontrase o único Porto do Estado, por onde é realizada toda entrada e saída de insumos via marítima.

Em Cabedelo/PB, atualmente 13.606 habitantes têm acesso à escola. A população economicamente ativa atinge $48,58 \%$ do total da população, tendo renda média de 1 (hum) salário mínimo. A população conta com uma rede de serviços de saúde que dispõe de um hospital geral e uma policlínica, e 19 unidades básicas de saúde, todos mantidos com recursos municipais e um hospital privado. As 19 equipes do PSF, assistiam 95\% da população, distribuídas em áreas que vão do centro à periferia.

No município foi criada a Coordenação de Saúde Mental, para iniciar o processo de organização da atenção à saúde mental. Através de estudos realizados pela Secretaria de Saúde do Município foi evidenciado que 512 pessoas faziam uso constante de medicação psicotrópica e que 1.050 pessoas estavam sendo atendidas pelo serviço ambulatorial de psiquiatria. Identificou- se também 87 pacientes egressos de Hospitais Psiquiátricos de João Pessoa (CABEDELO, 2003). Foi visto também que a reinternação ainda é uma prática constante. Para enfrentar essa situação, o município implantou em 2004, um Centro de Atenção Psicossocial - CAPS I e 2 leitos de psiquiatria no hospital geral.

\section{Sujeitos do estudo}

A pesquisa foi realizada com 4 (quatro) enfermeiras das unidades básicas de saúde do município de Cabedelo/PB e que estavam em atuação, por um período mínimo de seis meses. A opção por esse período decorre do entendimento de que nesse intervalo, a enfermeira já tem condições de adquirir informações básicas a respeito do município e da população atendida na área de abrangência de sua UBS.

\section{Aspectos Éticos}

A pesquisa foi aprovada pelo Comitê de Ética e Pesquisa do Centro de Ciências da Saúde - CCS da UFPB, obedecendo a Resolução 196/96 do Conselho Nacional de Saúde - CNS, do Ministério da Saúde - MS, que trata da pesquisa envolvendo seres humanos.

\section{Produção do material empírico}

Para a obtenção do material empírico foi utilizada a técnica de entrevista semi-estruturada as quais foram gravadas e posteriormente transcritas em forma de texto. A produção do material empírico foi realizada no mês de abril do ano de 2004.

\section{A análise do material empírico}

O material foi analisado pela técnica de análise do discurso proposta por Fiorin (1998) para o qual o texto é uma produção material que permite a identificação das visões de mundo dos sujeitos. Ele é histórico porque seu sentido é dado, também, por suas relações com o exterior, com as concepções que existem na sociedade da época em que ele foi produzido.

Os textos foram lidos exaustivamente e decompostos em fragmentos que foram agrupados por temas coincidentes e divergentes sobre o que 


\section{DISCUSSÃO DO MATERIAL EMPÍRICO}

A análise do material permitiu a construção das seguintes categorias temáticas: $O$ trabalho de Enfermagem como assistência/cuidado de saúde; a concepção do processo saúde-doença mental orientando as ações da práxis.

\section{O trabalho de Enfermagem como assistêncial cuidado de saúde}

$\mathrm{Na}$ análise das concepções dos entrevistados sobre o trabalho de enfermagem, desenvolvido no PSF, identificamos a compreensão do trabalho associada ao tema assistência/cuidado de saúde e à categoria "assistência, como atividade para atender a demanda do referido Programa", conforme os depoimentos:

Meu trabalho consiste na assistência à família como um todo. Aqui a gente tem os programas de hipertensão, diabetes, gestante, saúde do idoso, a saúde da mulher. Temos também a atenção à criança, como um todo, criança de 0 a 5 anos a gente dá assistência, a saúde como um todo (E1).

A Enfermagem como profissão da área de saúde, tem buscado historicamente a construção do cuidar como seu objeto de trabalho cuja abordagem teórica defende um cuidar planejado, contextualizado e em articulação com as tecnologias disponíveis na contemporaneidade. Para sua efetivação, precisa assumir seu compromisso com a mudança e manifestar na sua prática a transformação assumida no plano do discurso.

Desse modo, "assistir como um todo" conforme o depoimento de E1, em análise, significa a aplicação de um conhecimento científico da profissão que, na atualidade, precisa materializar sua compreensão, sobre o novo modelo de assistência que o SUS manifesta nos princípios filosóficos, sintetizados nos significados apontados nas categorias, Universalidade, Eqüidade e Integralidade. A abrangência dos sentidos dessas categorias orientadoras do SUS tem um efeito no campo das políticas que precisa ser visualizado, também, no âmbito do trabalho, na dimensão das práticas profissionais para a superação paradigmática pressuposta pelo movimento social da Reforma Sanitária. Nesse sentido, a compreensão de Integralidade defendida por Cecílio (2001) designa a assistência por meio do cuidado integral que refere tanto a promoção quanto à recuperação da saúde física e mental.

Uma das contradições produzidas no trabalho para a efetivação SUS, por meio dos Programas Saúde da Família é precisamente a não inclusão das ações de saúde mental imediatamente à sua constituição, quando o novo modelo reproduziu um aspecto do modelo tradicional de assistência, a dicotomia mente/ corpo, pela não consideração da área da saúde mental como uma de suas prioridades.

O depoimento em análise realça o modo como as enfermeiras organizam seu trabalho dentro do serviço, para atender às demandas da população e as do Ministério da Saúde, determinadas pelos Programas de Saúde do Idoso, da Mulher e da Criança.

Desse modo, os elementos do processo de trabalho de enfermagem refletem um momento histórico de transição na área da saúde, quando a ruptura com o modelo antigo ainda não aconteceu porque os pilares de sustentação da categoria Modelo de Assistência ainda não sofreram mudanças transformadoras de modo articulado, sendo o processo de trabalho o pilar que oferece mais resistência, precisamente por ser ele o pilar de maior potencial transformador e de maior visibilidade da transformação/conservação.

Ao analisar os depoimentos dos enfermeiros do PSF, no cenário deste estudo, verificamos uma freqüente recorrência dos temas que se referem aos processos de trabalho assistir (Cuidar/Fazer) tanto no enfoque Individual como no Coletivo conforme os depoimentos:

O que mais assistimos são as doenças respiratórias agudas: Crianças com pneumonia, bronquites, problemas respiratórios. Crianças com diarréias, a gente presta a assistência, mas, ela volta para casa e se re-contamina, pois, suas residências não têm saneamento (E1).

Prestamos assistência à saúde do adulto. É alto o número de diabéticos, alcoolismo, hipertensão e violência doméstica por causa do alcoolismo, do uso de droga por adolescentes (E2).

Verifica-se que o trabalho de enfermagem é 
desenvolvido na abordagem da Saúde Coletiva, no enfoque individual, conforme o primeiro depoimento que se refere a um objeto da Saúde Coletiva, o saneamento básico. Esse tema remetenos ao saber da Educação Sanitária como instrumento do trabalho. O processo de Assistir/ Cuidar individual tem como objeto, as doenças, inclusive as doenças mentais; como instrumento, apresenta-se o saber instrumental da Medicina Preventiva e da Psiquiatria Preventiva; a finalidade, além da cura, manifesta-se a Prevenção, a Educação em Saúde e a Vigilância Sanitária. Desse modo, mesmo na perspectiva da ampliação da atenção básica, confirma-se o que Antunes (2003) afirma, quanto a visão brasileira dos serviços de saúde no sentido da implantação do SUS, isto é, mantém o conservadorismo das ações programáticas, reproduzindo-se a lógica fragmentada, departamentalizada, e, não a integralidade, preconizada pelo SUS.

Observou-se, portanto, um trabalho que ainda preserva aspectos do modelo tradicional da Saúde Pública, mas incorpora novos aspectos da proposta da Saúde Coletiva, em um movimento de transformação que revela um momento de transição, pela incorporação de novos instrumentos de trabalho, condição necessária a um novo modelo que contemple a saúde mental.

A compreensão dos pressupostos do movimento pela Reforma Psiquiátrica, suas diretrizes, e uma educação permanente da força de trabalho são imprescindíveis para a transformação do processo de trabalho no sentido da efetivação do SUS, sobretudo no que se refere à integralidade da assistência. A transformação dessa complexidade em ações práticas, em trabalho de saúde/saúde mental é um dos grandes desafios dos profissionais de saúde, entre os quais os enfermeiros.

\section{A concepção do processo saúde-doença mental orientando as ações da práxis}

A partir da análise do material empírico, foi possível identificar a concepção processo saúde doença mental, bem como os elementos do processo de trabalho: o objeto, o instrumento e a finalidade, que orientaram o trabalho dos entrevistados. Os discursos convergiram para o reconhecimento de um tema: saúde/doença como estados de equilíbrio/desequilíbrio do organismo.

As concepções do processo saúde doença mental expostas pelos sujeitos discursistas, atendem ao modelo clínico individual, organicista e biológico na maneira de conceber a doença mental, contrapondo-se ao modelo de atenção psicossocial, bem como à atual política de organização dos serviços de saúde na perspectiva da Reforma Psiquiátrica, onde a atenção básica deve ser realizada dentro das unidades básicas de saúde. Isso pode ser visto nos depoimentos a seguir:

A médica é responsável por algo que apareça de saúde mental. A gente somente acompanha realizando visitas (E4).

A gente não aborda muito a saúde mental. Quando aparece algo, só quem faz alguma coisa é a médica. Ela prescreve e a enfermagem só acompanha. Mas é só isso. Eu não me identifico com essa área. A gente aqui trabalha mais na saúde da mulher (E2).

Nos depoimentos acima, observa-se que ainda há uma exclusão da área de saúde mental na atenção básica. As enfermeiras consideram que o atendimento a saúde mental restringe-se a prescrição de medicamentos e que cabe a elas apenas o acompanhamento. Chamamos a atenção para o fato de que não se deve considerar a saúde mental como um programa de distribuição de medicamentos psicotrópicos. Compreendemos que as ações de saúde mental, perpassam transversalmente todos os cuidados gerais de saúde em todos os grupos etários. Cuidar do doente mental é uma outra questão, pois envolve um conhecimento especial, porém não exclusivo.

A concepção de doença mental para desenvolver o trabalho, do modo como vem se processando, segundo o discurso das enfermeiras, tem como base, o saber como instrumento do trabalho, advindo da psiquiatria tradicional, a partir da idéia de doença mental como noção de periculosidade, necessitando, portanto, da medicalização da doença como controle de condutas indesejáveis. Assim, reproduz-se a abordagem medicalizante, imprimindo seu caráter predominantemente conservador ao trabalho em saúde mental, conforme o depoimento: 
Quando tem um caso como o de[...] a gente intervém, porque a mãe não queria nem internar, a gente internou porque ela não podia ficar no meio da rua, tirando a roupa, totalmente descontrolada, foi quando a gente levou para a Secretaria e conseguimos o internamento dela, mas a mãe não queria não, e tirou ela logo, e continuou o tratamento em casa com o médico mesmo, o acompanhamento dela foi feito com o médico de lá do hospital mesmo e a médica daqui só fazia o controle do psicofármaco [...] (E4).

Nesse depoimento verificamos a importância que os profissionais de saúde ainda atribuem ao hospitalocentrismo, pois, mesmo com a disposição da família em manter seu parente em tratamento psiquiátrico em domicílio, a enfermeira insistiu na hospitalização de modo compulsório, em uma prática de tendência oposta às diretrizes da Reforma Psiquiátrica. Esta tem como base filosófica a inclusão social por meio da desinstitucionalização, o que significa a negação do hospital psiquiátrico tradicional como instrumento terapêutico.

Os modos de exclusão social das sociedades capitalistas têm em comum a perda das relações sociais, e o combate à exclusão social passa pelo reconhecimento dos direitos de cidadania do excluído (DEMO, 1998). As sociedades capitalistas produzem obstáculos que impedem a inclusão do portador de doença mental, que deve ser manifestada, segundo Silva (2003) na libertação da tutela dos portadores de doenças mentais, significando dizer que é necessário uma revisão dos saberes que sustentam a psiquiatria tradicional, bem como do próprio processo de trabalho das equipes de saúde mental, no sentido de se conseguir a finalidade pretendida.

A visão tradicional apresentada nos discursos, desta investigação, aponta a necessidade de reformulação dos processos de trabalho em enfermagem, pois, sua prática pautada na ideologia dominante, evidencia uma distância da superação da assistência tradicional, requerendo novas formas de pensar e agir em relação ao processo saúde-doença mental. A posição social dos sujeitos participantes deste estudo, no que diz respeito ao processo saúdedoença mental, emerge em seus discursos e são reflexos de um sistema de saúde hegemônico e conservador.

Evidenciamos que as práticas dos enfermeiros entrevistados são pautadas por saberes que desconsideram a complexidade da assistência à saúde mental e, nesse enfoque, o processo de trabalho de enfermagem não se apresenta relacionando a desinstitucionalização, finalidade da Reforma, com a finalidade do trabalho, para, assim, superar o modelo asilar e excludente. Nesse caso, o PSF deve se apresentar com o caráter substitutivo, através de novas práticas de assistência centrada em um novo processo de trabalho, voltados para as necessidades individuais e sociais e não somente para as demandas. Assim, entre as contradições existentes na atuação profissional da Enfermagem a serem superadas, uma das mais complexas e de maior urgência é a que se produz entre o saber e a prática profissional e ela se reflete diretamente nos processos de trabalho.

Nos contextos dos novos serviços da atenção básica, cujas estratégias de intervenções deveriam buscar "privilegiar a dimensão coletiva do processo saúde doença, através de modelos interativos que incorporam ações individuais e coletivas", o enfoque tradicional não permite que se compreenda o processo saúde doença, mas somente a enfermidade resumida através de sinais e sintomas (OLIVEIRA; EGRY, 2000, p.13).

Assim, os elementos do processo de trabalho que orientam tais práticas apresentam como objeto a doença mental, como finalidade, o seu equilíbrio ou cura, e os instrumentos utilizados são os saberes da psiquiatria tradicional, em que a assistência de enfermagem tem como finalidade promover a readaptação do comportamento da pessoa com doença mental e o seu controle no espaço hospitalar. Desse modo, a concepção do processo saúde-doença mental da psiquiatria hegemônica orienta a escolha dos demais elementos do processo de trabalho determinando seu caráter excludente e segregador.

\section{CONSIDERAÇÕES FINAIS}

As concepções dos enfermeiros sobre o processo de trabalho desenvolvido no PSF, bem como as características do mesmo, considerando 
que o processo saúde-doença é o norteador das ações na atenção básica, revelaram que o trabalho desenvolvido pelos enfermeiros, sujeitos do estudo, é compreendido como o assistir, tendendo para o cuidado que tem a doença como objeto de trabalho, preservando aspectos do modelo tradicional da Saúde Pública. Contudo, esses profissionais vêm incorporando em seu processo de trabalho novos aspectos da proposta da Saúde Coletiva, em um movimento no sentido superação paradigmática. Compreendemos que esse momento de transição requer um novo processo de trabalho, que se manifesta nas propostas da Reforma Psiquiátrica, e que esta é uma realidade desejada na maioria dos municípios brasileiros, mas ainda não vivenciada como práxis, como transformação do modelo de assistência psiquiátrica tradicional, mas como uma aproximação que permite pensar a superação paradigmática como um horizonte de possibilidade a ser perseguido.

Desse modo, a concepção dos enfermeiros sobre o processo saúde doença mental, ainda carece de articulação com as condições sociais, econômicas e culturais, na qual o ser humano pode ser compreendido na dinâmica contraditória da realidade social, pois, para os enfermeiros em questão, objeto de trabalho ainda é a doença mental, a finalidade anunciada de inclusão social no plano da política coexiste com a busca pela cura da doença mental, e os instrumentos são os saberes da Psiquiatria Tradicional que norteiam as práticas, pautada no paradigma da Saúde Pública. Desse modo, os elementos que constituem o processo de trabalho se apresentam desarticulados com a nova formulação política e organizacional dos serviços de saúde, hoje representada pelo PSF, e que deve corresponder a um novo processo de trabalho, cujos elementos apresentem-se com potencial transformador da realidade das pessoas com doença mental.

A atual política nacional de saúde mental tem anunciado seu interesse na municipalização das ações de saúde mental de base comunitária, o que constitui a possibilidade de avanço no sentido da superação do modelo asilar e excludente. Por outro lado, a efetivação desse interesse, ainda é inexpressivo porque há uma desarticulação entre a rede de cuidados de saúde e particularmente da saúde mental. No município em questão, não foram desenvolvidos os mecanismos de referência e contra referência, e falta uma articulação entre outros setores para se implantar uma rede de apoio psicossocial. Apesar de se constatar os esforços por parte de técnicos envolvidos com a atenção básica de saúde, e dos docentes que atuavam no município, percebe-se que ainda não se desenvolveram forças suficientes que possibilitem uma ruptura com o modelo tradicional.

O PSF ainda não provocou mudanças efetivas em direção ao seu propósito teórico. Sua proposta é relevante, visto que se busca superar o paradigma sanitário imposto, em uma ampliada produção social da saúde. No entanto, é necessário um suporte maior no que concerne a produção de saberes, no sentido de estimular a participação mais reflexiva, tanto dos profissionais envolvidos, quanto da população.

O estudo também aponta a possibilidade de superação de impasses e limites, que se constituem em contradições, para subsidiar avanços na atenção em saúde mental do município, através de uma (re) estruturação da política municipal de saúde mental que favoreça a educação permanente dos enfermeiros e dos outros profissionais envolvidos no trabalho do PSF (FRAGA, 2003).

ABSTRACT: Study carried out with the objective to describe the conceptions of the nurses who act in the "Family Health Program"PSF of the city of Cabedelo about the process of nursing work, the characteristics of this process, and, to identifythe aspects of the process health-illness that these nurses approach with greater and minor frequency in daily of the practical professional. It had theoretical referencial in the models of assistance to health introduced in Brazil, in the strategy of the PSFand in the process of work in Mental Health, the metodological referencial had inspiration the Materialism Historical and Dialectical. The empirical material was collected by means of interviews and analyzed through the technique of analysis of the speech. The analysis allowed to identify the knowledge and the practical ones that guide the investigated work, assisting the citizens of the inquiry to develop actions of nursing capable to subsidize and approach with the overcoming of the O 
direction of the social inclusion of the person with insanity. The PSF is a strategy for the accomplishment of the only System of Health -SUS and such as a tool that can be used in the direction of gathering the actions of mental health approaching this specific area with SUS and allowing the advance of the movement for the Psychiatric Reform in the perspective of one of its guidelines, the desinstitutionalization of the illness and the person with insanity.

KEY WORDS: Nursing; Family health program (PSF); Mental health.

\section{REFERÊNCIAS}

ANTUNES, M. J. Saúde: Direitos de todos e dever do estado? Programa saúde da Família: Filosofia, diretrizes e realidade. A presença da mulher no controle social das políticas de saúde: anais da capacitação de multiplicadoras em controle social das políticas de saúde. Rede Feminista de Saúde: Belo Horizonte: Mazza Edições, 2003. 102-122.

BRASIL. Incentivo à participação popular e controle social no SUS. Textos técnicos para conselheiros de saúde. Brasília, DF, 1994.

CABEDELO, Relatório sobre saúde mental no município de Cabedelo. Coordenação de Saúde Mental; 2003, Cabedelo, 2003.

CECÍLIO, L. C. de O. As necessidades de saúde como um conceito estruturante na luta pela integralidade e equidade na atenção em saúde. In: PINHEIRO, R.; MATTOS, R. A. de (Org). Os sentidos da Integralidade na atenção e no cuidado à saúde. Rio de Janeiro: ABRASCO, 2001.

DEMO, P. Avaliação qualitativa. São Paulo: Cortez, 1998 (Coleção polêmicas do nosso tempo).

FIORIN, J. L. Linguagem e ideologia. 6 ed. São Paulo: Ática, 1998.

FRAGA, M. N. de O. Inserção de ações de saúde mental a atenção básica de saúde. Texto elaborado em fevereiro de 2003 como subsídio a módulo de acompanhamento a profissionais vinculados ao Programa de Interiorização do Trabalho em Saúde(PITS) e atuantes em municípios do Ceará.

IBGE - Instituto Brasileiro de Geografia e Estatística. Censo Demográfico 2000, tabela população residente, 2000. Disponível em : <http: www.ibge.gov.br/

KONDER, L. O futuro da filosofia da práxis. $O$ pensamento de Marx no século XXI. São Paulo: Paz e Terra, 1992.

LOWY, M. Ideologias e ciências sociais: elementos para uma análise marxista. 5 ed. São Paulo: Cortez, 1989.

MATTOS, R. A. de Os sentidos da Integralidade: algumas reflexões acerca de valores que merecem ser defendidos. In: PINHEIRO, R.; MATTOS, R. A. de (Org). Os sentidos da Integralidade na atenção e no cuidado à saúde. Rio de Janeiro: ABRASCO, 2001.

OLIVEIRA, M. A. C.; EGRY E. Y. A historicidade das teorias interpretativas do processo saúde doença. Rev. Esc. Enf. USP, v. 34, n. 1, p. 9-15, mar 2000.

SILVA, A. T. M. C. A reforma psiquiátrica em João Pessoa: Limites e possibilidades de transformação do trabalho para produzir saúde mental. (Tese de doutorado): Escola de enfermagem da USP, 2003.

TORREZ, M. N. F. B.; BARROS, L. R.; GOULART, V. M. P. A educação profissional de nível técnico e a estratégia Saúde da Família: renova-se o desafio. Rev. Bras. Enf., v. 53 nº especial, p. 61-9, 2000.

ENDEREÇO DOS AUTORES: Rua Pedro de Franca Macedo, 451, apto 103B. Bancários. 58051-540 João Pessoa/PB khiviakiss@click21.com.br 